EM

\title{
REFORMA DEL SECTOR PÚBLICO DE SALUD EN LA PROVINCIA DE MISIONES (ARGENTINA) Y SU IMPACTO EN LA ATENCIÓN EN LA FRONTERA POSADAS-ENCARNACIÓN ${ }^{1}$
}

\section{REFORM OF THE PUBLIC HEALTH SECTOR IN THE PROVINCE OF MISIONES (ARGENTINA) AND ITS IMPACT ON CARE IN THE FRONTIER POSADAS-ENCARNACIÓN}

\author{
REFORMA DO SETOR PÚBLICO DE SAÚDE NA PROVÍNCIA DE \\ MISIONES (ARGENTINA) E SEU IMPACTO NA ATENÇÃO A \\ FRONTEIRAS POSADAS-ENCARNACIÓN
}

Florencia ALMÚA ${ }^{2}$

\begin{abstract}
Resumen: En las últimas décadas, los procesos de reforma del Estado encarados en América Latina y particularmente en Argentina se han profundizado, buscando la racionalización y modernización del aparato estatal, con el objetivo de construir un Estado más eficaz y eficiente. La región fronteriza analizada comprendió a Itapúa (Paraguay), con su re-estructuración histórica del sistema de Salud Pública Nacional y un avance dispar en cada distrito; y, por otra parte, a Misiones (Argentina), quien lleva adelante un proceso de modernización de su sistema, con la incorporación de infraestructura de alta complejidad. El objetivo del presente trabajo consistió en analizar una de las principales iniciativas de reforma del sector de la salud pública implementada en uno de los Hospitales de referencia de dicha región, a través de la implementación de algunas herramientas del Nuevo Management Público o Gestión por Resultados. El Hospital Escuela de Agudos Dr. Ramón Madariaga, ubicado en la Provincia de Misiones, viene desarrollando en los últimos años una transición desde el modelo de Gerencia Pública Tradicional hacia el Nuevo Management Público. Esta reforma ha permitido aumentar la capacidad y calidad de los servicios, recibiendo derivaciones no solo de pacientes nacionales, sino de toda la región, incluida la frontera.
\end{abstract}

Palabras claves: Servicios de Salud - Frontera - Estado - Management Público

\begin{abstract}
In recent decades, the processes of state reform faced in Latin America and particularly in Argentina have deepened, seeking the rationalization and modernization of the state apparatus, with the aim of building a more effective and efficient State.The border region analyzed included Itapúa (Paraguay) with its historical restructuring of the National Public Health system and a disparate progress in each local district; and, on the other hand, Misiones (Argentina) who carries out a process of modernization of its system, with the incorporation of highly complex infrastructure. The objective of this work was to analyze one of the key initiatives of reform of the public health sector implemented in one of the reference hospitals of the region, through the implementation of some tools of the New Public Management or Management for Results. Dr. Ramón Madariaga School Hospital, located in the Province of Misiones, has been developing in recent years a transition from the Traditional Public Management model to the New Public Management. This reform has allowed increasing the capacity and quality of services, receiving referrals not only from national patients, but from the entire region, including the frontier.
\end{abstract}

\footnotetext{
${ }^{1}$ Compilado de Trabajos presentados (adaptado) en el IV GEOFRONTEIRAS de 2017 (UFGD - Dourados) y V SEMINARIO INTERNACIONAL DE LOS ESPACIOS DE FRONTERA (V GEOFRONTERA): Territorialidades y Sujetos transfronterizos (Posadas, Misiones, Argentina).

${ }^{2}$ Licenciada en Economía. Email: florencia_almua@hotmail.com
} 
EM

QUESTÃO

V.13 N. $02 \downarrow 2020$

pág. 164-177

Key-words: Health services - Frontier - State - Public Management.

Resumo: Nas últimas décadas, aprofundaram-se os processos de reforma do Estado enfrentados na América Latina e, em particular, na Argentina, buscando a racionalização e modernização do aparato estatal, com o objetivo de construir um Estado mais eficaz e eficiente. A região de fronteira analisada incluiu a Itapúa (Paraguay), com sua reestruturação histórica do Sistema Nacional de Saúde Pública e um progresso díspar em cada distrito; e, por outro lado, Misiones (Argentina), que realiza um processo de modernização de seu sistema, com a incorporação de infraestrutura altamente complexa. O objetivo do presente trabalho foi analisar uma das principais iniciativas de reforma do setor de saúde pública implementadas em um dos hospitais de referência da região, através da implementação de algumas ferramentas da Nova Gestão Pública ou Gestão de Resultados. O Hospital Escuela de Agudos Dr. Ramón Madariaga, localizado na Província de Misiones, vem desenvolvendo nos últimos anos uma transição do modelo de Gestão Pública Tradicional para a Nova Gestão Pública. Essa reforma permitiu aumentar a capacidade e a qualidade dos serviços, recebendo encaminhamentos não apenas de pacientes nacionais, mas de toda a região, inclusive da fronteira.

Palabras claves: Serviços de saúde - Fronteira - Estado - Gestão Pública.

\section{Introducción}

A partir de la Segunda Fase de Reforma del Estado, iniciada en la Argentina en el año 1996, la Administración Pública Nacional ha estado llevando adelante interesantes transformaciones (ESSAYAG, 2000, p. 1). Las mismas obedecen a procesos de racionalización y modernización del aparato estatal, con el objetivo construir un Estado más eficaz y eficiente. En este sentido, Essayag (2000) se sostiene que "modernizar al Estado significa hacer que su gestión, en lugar de basarse exclusivamente en procedimientos administrativo-burocráticos, se oriente hacia la búsqueda de resultados rindiendo cuentas por ellos".

Siguiendo a Góngora y Spadafora (2003), los hospitales necesitan ser más efectivos y eficientes, siendo necesario que se produzcan profundos cambios en los servicios públicos de salud, dado que "sus estructuras burocráticas, rígidas y verticales afrontan limitaciones legales y centralistas en la gestión de sus recursos financieros y humanos, y su cultura institucional es muy poco sensible a las necesidades de las personas y a los cambios de entorno." (GÓNGORA Y SPADAFORA, 2003, p. 23).

El análisis queda acotado a las ciudades fronterizas del MERCOSUR: Posadas (Misiones, Argentina) y Encarnación (Itapúa, Paraguay). Esta región se caracteriza por su economía marginal, por su alta dependencia del sector primario, creciente migración ruralurbana, bajo nivel de capitalización y exposición a la competencia regional asimétrica basada en la informalidad y la ilegalidad (contrabando y tráficos ilegales varios) (VELAZCO, 2000).

El marco teórico de referencia abarca el camino hacia Nuevo Management Público (Gestión por Resultados), que buscaría reemplazar características de la administración pública tradicional, tomando prestadas diversas ideas de la administración privada, y sosteniendo como sus valores esenciales los conceptos de: clientes, gerentes, flexibilidad, riesgo, creatividad, evaluación de resultados, privatizaciones o tercerizaciones (ESTÉVEZ, 2001).

En este marco de grandes transformaciones, analizamos de qué manera algunas herramientas de la gestión por resultados se han implementado en uno de los Hospitales de referencia en la zona fronteriza: el Hospital Escuela de Agudos Dr. Ramón Madariaga, ubicado en la Provincia de Misiones. Éste se halla desarrollando en los últimos años una transición desde el modelo de Gerencia Pública Tradicional hacia el Nuevo Management Público. Esta reforma ha permitido aumentar la capacidad y calidad de los servicios, 
EM

recibiendo derivaciones de pacientes no solo de la provincia de Misiones, sino de toda la región, incluida la frontera.

Una de las consecuencias de la modernización del sector salud de los últimos años se ha visto reflejada en el aumento de consultas de pacientes nacionales, pero también los extranjeros que cruzan la frontera en búsqueda de servicios sanitarios de calidad. Cabe aclarar que, si bien el vecino país de Paraguay viene atravesando en los últimos años una reestructuración histórica de su sistema de Salud Pública Nacional, aún se observa un avance dispar en cada distrito y un camino por recorrer en pos de poder poner a disposición del paciente paraguayo un servicio de salud eficiente y de calidad, sobre todo en lo referido a las prestaciones de alta complejidad.

\section{La Gerencia Pública Tradicional (GPT) vs. El Nuevo Management Público (NMP)}

Siguiendo a Estévez y Blutman (2005) se pueden distinguir tres tipos principales de programas de Reforma: weberianas, de responsabilización (accountability) o gerenciales.

Las reformas gerenciales o el NMP sería el nuevo ethos o cultura organizacional que apunta a formar un comportamiento cultural común en los miembros de la administración pública, buscando reemplazar al viejo que animaba a la administración pública tradicional.. Fue utilizado como instrumento de cambio en las administraciones de los países desarrollados y su advenimiento coincide con la asunción de Margaret Thatcher como primer ministro de Gran Bretaña en 1979.

El modelo anterior estaba estructurado por ideas como: ciudadanía, comunidad, equidad social, transparencia, interés público, evaluación de procesos, prudencia, ética, etc. En cambio, el NMP toma prestadas diversas ideas de la administración privada, y sostiene como sus valores escenciales los conceptos de: clientes, gerentes, flexibilidad, riesgo, creatividad, evaluación de resultados, privatizaciones o tercerizaciones (ESTÉVEZ, 2001: 5).

Desde un punto de vista gerencial, el objetivo del NMP, es el mejoramiento de la calidad de los servicios producidos por las instituciones públicas, por medio del incremento de la participación de los ciudadanos, quienes serían los primeros beneficiarios de los precitados servicios.

Siguiendo a Estévez (2001) se pueden analizar las diferencias entre el NMP y la Administración Pública tradicional de acuerdo a:

Foco - Mientras que en la Gerencia Pública tradicional, el centro de la acción se dirigía hacia el ciudadano o la comunidad, para el NMP el cliente será el eje de su preocupación. Se sustituye al ciudadano por el cliente; es decir una categoría política es reemplazada otra ligada al mercado. En el NMP, el cliente exige al funcionario una cierta calidad del servicio público.

Principales Recursos o útiles - Con el NMP, se pone el acento sobre la gestión (mediante técnicas de management moderno), más que sobre la administración de procesos de implementación de políticas públicas, como era el caso de la Gerencia Pública tradicional. El NMP enfatiza sobre el mejoramiento de las prácticas gerenciales, mediante la modelización estadística, o las técnicas analíticas para modelizar o redefinir los procesos públicos. El NMP pone el acento sobre los resultados y no sobre los procesos, como la GPT.

Características de los Funcionarios Públicos - El NMP exige funcionarios emprendedores, valorando su capacidad de accionar; en oposición al analista de la GPT. El NMP le da al funcionario un margen de maniobra mayor, porque se pretende que sea emprendedor, que tome riesgos, que negocie con sus clientes, y que sea un hombre de acción más que un analista. El funcionario emprendedor debe ser recompensado por su creatividad y entusiasmo. El NMP pone el acento sobre los gerentes más que sobre los empleados. 
EM

Los Valores: en la Gerencia Pública Tradicional priman la prudencia, la estabilidad en el puesto de trabajo, la antigüedad, la justicia, entre otros; en tanto en el NMP se manejan espíritus emprendedores, con libertad de acción para los managers, flexibilidad, creatividad, entusiasmo, propensión al riesgo, etc.

Vocabulario - En el NMP se manejan los conceptos de: servicios a los clientes, calidad, habilidades, gerenciamiento, fortalecimientos institucionales, etc.; en tanto en el Gerencia Pública Tradicional el interés público o general, la democracia, la equidad social, el debido proceso, los reglamentos, etc. siguen siendo los conceptos que rigen su accionar.

Cultura Organizacional - es el conjunto de normas, creencias, valores, costumbres, rituales, lenguajes, artefactos y presunciones básicas existentes en una organización y que afectan a su desarrollo $=>$ Gerenciamiento de tipo privado o comercial (NMP) vs. Burocrática jerárquica (GPT).

Estructuras - La forma en que se estructura una organización tiende a mostrar cómo los RRHH son distribuidos teniendo en cuenta las relaciones interfuncionales. La estructura permitirá indicar las líneas de responsabilidad, autoridad y comunicación formal $\Rightarrow$ División horizontal o funcional del trabajo (NMP) vs. División vertical o jerárquica del trabajo (GPT).

Considerando que el modelo de Gestión por Resultados (GPR) está inspirado en la visión gerencial de la gestión pública, Estévez (2003) lo resalta como un instrumento de gestión de las organizaciones que: 1) Estructura la gestión en torno al proceso resultadosproductos-recursos; 2) Descentraliza la toma de decisiones y flexibiliza el uso de los recursos; 3) Relaciona el planeamiento estratégico, el proceso presupuestario, el monitoreo de gestión y la rendición de cuentas por resultados; y 4) Se caracteriza por el predominio de acciones creativas y de rutinas de calidad.

La gestión por resultados incorpora la planificación estratégica en la determinación de la misión, la estrategia y los objetivos de la institución. Incluye, además, el diseño de un sistema de incentivos selectivos positivos y negativos para el desempeño de sus recursos humanos y organiza un sistema de control de la gestión validado a través de indicadores cualicuantitativos (ESSAYAG, 2000).

\section{Un caso de aplicación en la zona de frontera: la transición del Hospital Escuela de Agudos Dr. Ramón Madariaga}

Según los historiadores Etorea y Freaza (2011), el 21 de enero de 1924 se inauguraba el nuevo Hospital Regional Común de Posadas y, en 1955, por decreto $\mathrm{N}^{\circ} 138$ cambia de nombre por el de "Dr. Ramón Madariaga". A partir del año 2005 se ponen en marcha las obras que dan comienzo al Nuevo Hospital Central Madariaga, con una inversión inicial de 90 millones de pesos. Para aquel entonces se esperaba que las nuevas instalaciones

[...], incrementen cualitativamente los servicios incorporando prestaciones al nivel d los grandes centros como ser: emergentología con capacidad para atender situaciones de catástrofe, diagnóstico por imagen, oncología, hematología, braquiterapia, hemodiálisis, unidad renal, centro de esterilización y centro de rehabilitación, terapia intensiva, unidad coronaria, quemados, aislados y adictos." (Discurso del Sr. Gobernador de la Provincia de Misiones, $1^{\circ}$ de Mayo 2008)

Es así como, en el año 2010 se habilita parte del Hospital Escuela de Agudos Dr. Ramón Madariaga, con una inversión que rondó los 140 millones de pesos. Comienza funcionando el servicio de diagnóstico por imágenes más moderno de toda la región, el sector 
EM

de emergencia y un piso de internación. El 8 de Julio del 2011 entra en vigencia la Ley que concibe el Parque de la Salud de la Provincia de Misiones "Dr. Ramón Madariaga" (LEY XVII - $\mathrm{N}^{\mathrm{o}}$ 70) y, en febrero del año 2012, su correspondiente Decreto Reglamentario $\mathrm{N}^{\circ}$ 83/12. Esta nueva figura combina el sector público de salud (en su carácter de Organismo Descentralizado, tiene dependencia funcional del Poder Ejecutivo por medio del Ministerio de Salud Pública) con el sector privado (el gobierno y la administración lo ejerce la Fundación Parque de la Salud Dr. Ramón Madariaga).

Esta decisión adoptada por el Gobierno Provincial significó un salto trascendental no solo en la cantidad y calidad de los servicios prestados, sino un cambio de paradigma en la Salud Pública de la provincia de Misiones. De esta manera es posible identificar en los mencionados instrumentos constitutivos, diferentes rasgos que dan cuenta del camino planteado hacia una nueva Gerencia Pública en el ámbito sanitario. Por un lado, si bien se exceptúa del pago solidario a las personas que no tienen seguro de cobertura asistencial (derecho-habientes), quedan bien definidos los "clientes": los ciudadanos con cobertura de obra social, las entidades de medicina prepaga, las compañías de seguro, empleadores en general, extranjeros sin residencia legal en el país y terceros obligados (Decreto 83/12 Anexo I). Respecto de los extranjeros sin residencia legal

[...], este cambio en la figura le otorga al paciente que cruza la frontera el derecho de recibir los servicios sanitarios pertinentes en las mismas condiciones que los pacientes nacionales, a través de una contraprestación. Así, por ejemplo, el paciente paraguayo, deberá ser visto ante la organización del hospital como cualquier otro ciudadano argentino que tenga obra social, siempre que realice el pago correspondiente. (ALMUA, 2016).

A partir de esta nueva concepción, se plantean los objetivos que apuntan no solo a lo asistencial (prevención, promoción, diagnóstico, rehabilitación y recuperación de la salud), sino también a la Docencia y Capacitación, al desarrollo del área Científica y a las actividades de Cooperación y Contribución con otras instituciones del Entorno. Se apuesta a un "modelo de gestión moderna y sustentable que tienda a la descentralización administrativa y se rija por el principio de eficiencia, que posibilite una óptima capacidad de resolución de las necesidades de las personas que requieran los servicios de salud" (LEY XVII - $\mathrm{N}^{\circ} 70$, art. $9^{\circ}$, inc. a). Así mismo, se resalta la obligación de constituir un "Plan Estratégico" a fin de proyectar las acciones necesarias y adecuadas de gestión y ejecución a fin de cumplir con los objetivos propuestos (LEY XVII - $\mathrm{N}^{\circ} 70$, art. $9^{\circ}$, inc. c).

En cuanto al recurso humano, la Ley garantiza la "sustentabilidad de la planta del personal profesional, no profesional existente y el necesario para cumplir con los objetivos propuestos" (Ley XVII - $\mathrm{N}^{\circ} 70$, art. 13ㅇ), mediante el desarrollo de tareas de formación, capacitación y perfeccionamiento constante de los mismos. Para todo ello, el Estado Provincial debe garantizar la autonomía financiera del Parque de la Salud de la Provincia de Misiones "Dr. Ramón Madariaga", asegurando en el Presupuesto General de la Administración Pública Provincial de cada año la autosuficiencia financiera necesaria para cumplir en forma eficiente los objetivos propuestos (LEY XVII - $\mathrm{N}^{\circ} 70$, art. $6^{\circ}$ ).

Es así como desde su puesta en marcha, el Gobierno de la Provincia de Misiones ha garantizado los recursos a fin de atender los gastos que específicos conforme a la Ley. El siguiente cuadro muestra la evolución del Presupuesto asignado al Parque de la Salud Dr. Ramón Madariaga en comparación al correspondiente al Ministerio de Salud de la Provincia: 
Tabla 1: Presupuesto del Parque de la Salud Dr. Ramón Madariaga

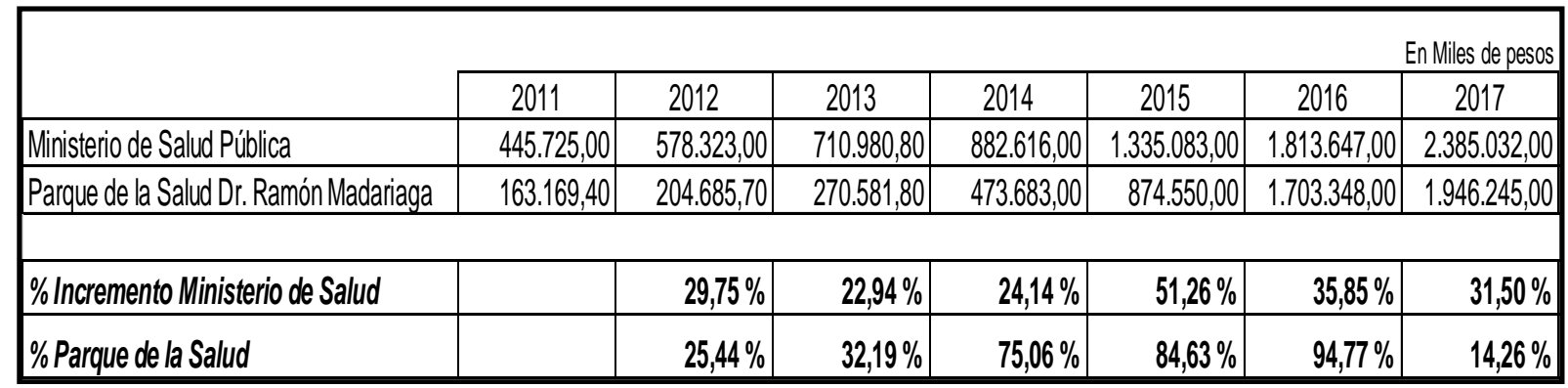

Fuente: Elaboración propia en base a Leyes Provinciales de Presupuesto Ejercicios 2011-2017

Esta reforma ha permitido aumentar la capacidad de servicios, recibiendo derivaciones de pacientes no solo de la provincia de Misiones, sino de toda la región, incluida la frontera.

Según datos tomados de la presentación hecha por el Ministro de Salud Pública de Misiones en la defensa de presupuesto 2018 ante la Comisión de Presupuesto en la Cámara de Representantes, el total anual de atenciones pasó de 410.147 en 2011 a 1.030 .557 en 2016, lo que significó un incremento de $151 \%$.

Una de las consecuencias de la modernización del sector salud de los últimos años se ha visto reflejada en el aumento de consultas de pacientes que cruzan la frontera en búsqueda de servicios sanitarios de calidad. Cabe aclarar que, si bien el vecino país de Paraguay viene atravesando en los últimos años una re-estructuración histórica de su sistema de Salud Pública Nacional, aún se observa un avance dispar en cada distrito y un camino por recorrer en pos de poder poner a disposición del paciente paraguayo un servicio de salud eficiente y de calidad, sobre todo en lo referido a las prestaciones de alta complejidad.

Otro de los aspectos que merece ser debidamente mencionado está relacionado al aumento significativo en los últimos años de las radicaciones "aparentes" de ciudadanos paraguayos, que en el afán de sentirse cubiertos por un seguro público de salud eficiente y gratuito, sientan residencia en argentina, pero regresan a vivir a su país (ALMÚA y ALIPRANDINI, 2015). Esta situación ha generado una distorsión en las estadísticas sanitarias, impidiendo disponer de información respecto de los servicios efectivamente prestados a los pacientes en la región de frontera argentino paraguaya.

Respecto de esta última situación, cabe aclarar que existe una discordancia entre el mencionado decreto y las legislaciones nacionales e internacionales (ALMÚA y ALIPRANDINI, 2015), pero que no es objeto de estudio del presente.

\section{Indicadores de producción sanitaria del Hospital Escuela de Agudos Dr. Ramón Madariaga}

Durante el periodo 2011-2016, los tres hospitales de alta complejidad de adultos de la Provincia de Misiones obtuvieron en total 149.079 Ingresos Hospitalarios. De dicho total, el 54,7\% correspondió al Hospital Escuela de Agudos Dr. Ramón Madariaga. Por su parte, para el mismo periodo se registraron en total 146.398 Egresos Hospitalarios, correspondiendo el $56,4 \%$, a mencionado efector. La Tabla 2 expone los principales indicadores sanitarios correspondiente al efector analizado durante el periodo 2011-2016: 
EM

QUESTÃO

V.13 N. $02 \downarrow 2020$

pág. 164-177

Tabla 2: Indicadores sanitarios Hospital Escuela de Agudos Dr. Ramón Madariaga - Posadas (Misiones, Argentina)

\begin{tabular}{|l|r|r|r|r|r|r|r|r|r|}
\hline & \multicolumn{1}{|c|}{2011} & \multicolumn{1}{c|}{2012} & \multicolumn{1}{c|}{2013} & \multicolumn{1}{c|}{2014} & \multicolumn{1}{c|}{2015} & \multicolumn{1}{c|}{2016} & \multicolumn{1}{c|}{2017} & Promedio & Variación \\
\hline Ingresos & 14.825 & 15.191 & 14.281 & 12.602 & 11.532 & 13.066 & 13.296 & 13.542 & $-10,31 \%$ \\
\hline Egresos & 14.752 & 15.180 & 14.756 & 12.700 & 12.160 & 13.050 & 13.480 & 13.725 & $-8,62 \%$ \\
\hline Giro de Cama & 72,8 & 64,5 & 55,6 & 44,3 & 45,2 & 47,2 & 42,1 & 53 & $-42,17 \%$ \\
\hline Dotación de camas & 165 & 241 & 248 & 285 & 264 & 264 & 264 & 247 & $60,00 \%$ \\
\hline N. ${ }^{\circ}$ de camas disponibles & 73.923 & 85.862 & 96.882 & 104.675 & 98.294 & 100.865 & 116.830 & 96.762 & $58,04 \%$ \\
\hline Paciente-día & 65.639 & 73.821 & 79.441 & 77.250 & 78.026 & 82.750 & 100.999 & 79.704 & $53,87 \%$ \\
\hline \% Ocuación de Camas & 88,8 & 86,0 & 82,0 & 73,8 & 79,4 & 82,0 & 86,4 & 83 & $-2,70 \%$ \\
\hline Promedio de Permanencia & & & & & & & & & \\
\hline Intervalo de Sustitución & 4,4 & 4,9 & 5,4 & 6,1 & 6,4 & 6,3 & 7,5 & 6 & $70,45 \%$ \\
\hline
\end{tabular}

Fuente: Elaboración propia en base a datos proporcionados por la Dirección de Programación y Planificación del Ministerio de Salud de Misiones, Argentina.

Respecto de las tendencias observadas, resulta merecedor destacar el descenso logrado a lo largo del periodo analizado tanto en los Ingresos como los Egresos Hospitalarios del Hospital Escuela de Agudos Dr. Ramón Madariaga. Este hecho se fundamenta en dos cuestiones claves:

Por un lado, la apuesta en la Política Pública de Descentralización de la Atención en Salud propuesta por el Estado Provincial a partir de la sanción de la Ley de Salud en 2007, básicamente a través del fortalecimiento de la Atención Primaria de la Salud. De esta manera se ha logrado, tanto a través de la inauguración de nuevos Centros de Atención Primaria de la Salud (CAPS) como de la re-adecuación de los hospitales niveles I y II, que las personas logren recurrir, en primera instancia, a estos efectores, descongestionando la atención de Alta Complejidad. Los CAPS, que son los efectores priorizados por la política de salud, en 2003 eran 43. Desde el 2003 al 2007 se priorizo la construcción de estos efectores a lo largo y ancho de la provincia, y al finalizar el 2007 se contaba con 298 Caps. Para el período 2007 al 2014 se inauguraron 73 CAPS, completando la red con 371 efectores.

Por otro lado, es necesario destacar que el Hospital Escuela de Agudos es el único de la provincia de Misiones que cuenta con un modelo de hospitalización alternativo: el servicio de internación domiciliaria. Éste consiste en prestar servicio exclusivamente a pacientes sin obra social en sus domicilios, con patologías que van desde el cumplimiento de antibióticos prolongados hasta el tratamiento y fines de vida con pacientes oncológicos pediátricos y adultos. Esta implementación viene a resolver significativamente el problema de ocupación hospitalaria, desarraigo del paciente y su familia, de la infección intrahospitalaria, etc.

Las estadísticas demuestran que en el periodo analizado disminuyeron los Ingresos Hospitalarios en un 10\%, pero a su vez, el número de pacientes-día se incrementó en un 54\%. Esto estaría indicando una mejora en las acciones resolutivas del efector, en tanto este último indicador representa al paciente que ingresa y egresa el mismo día.

Puede observarse, además, que entre los años 2011 y 2017 la dotación de camas y el número de camas disponibles del Hospital Escuela de Agudos se incrementó en un $60 \%$ y $58 \%$, respectivamente. El crecimiento se acentúa en los cuatro primeros años de su puesta en marcha, donde crece entre el 2011 y el 2014 en 41,6\%, pasando de 73.923 a 104.675 camas disponibles, respectivamente. Esto está asociado básicamente a la inauguración y habilitación de los diferentes sectores del efector.

En cuanto al Promedio de Permanencia, se calcula como el cociente entre el total de pacientes-día en un periodo respecto del total de egresos en el mismo periodo. Considerando 
que el resultado estándar esperado para los Hospitales Nivel III es de 9 días, puede observarse que el hospital analizado se encuentra por debajo de dicho promedio. No obstante, ha experimentado un incremento del $42.5 \%$ en el promedio de días de permanencia durante los años 2011-2017, pasando de un promedio de permanencia de 4,4 días en 2011 a 7,5 días en 2017. Respecto a las Consultas médicas ambulatorias registradas año 2017, el Hospital Escuela registró un total de 237.348, de las cuales un 56\% (132.843) correspondieron a Consultorios Externos y el restante a consultas en Guardias.

Tabla 3: Consultas médicas ambulatorias registradas año 2017. Hospital Escuela de Agudos Dr. Ramón Madariaga. Posadas (Misiones, Argentina)

\begin{tabular}{|l|r|r|r|}
\hline & \multicolumn{1}{|c|}{2017} & Total Zona & \multicolumn{1}{c|}{$\begin{array}{c}\% \\
\text { Participación }\end{array}$} \\
\hline Consultorio Externo & 132.843 & 328.423 & $40,45 \%$ \\
\hline Guardia & 104.505 & 240.080 & $43,53 \%$ \\
\hline Total Efector & 237.348 & 568.503 & $41,75 \%$ \\
\hline
\end{tabular}

Fuente: Elaboración propia en base a datos proporcionados por la Dirección de Programación y Planificación del Ministerio de Salud de Misiones, Argentina.

El total de atención médicas se incrementó entre el 2011 y 2017 en un 202\%, pasando de 410.147 atenciones a 1.237.506.

Tabla 4: Evolución de las atenciones médicas. Hospital Escuela de Agudos Dr. Ramón

Madariaga. Posadas (Misiones, Argentina)

\begin{tabular}{|c|r|}
\hline Año & $\begin{array}{c}\text { Total de } \\
\text { atenciones }\end{array}$ \\
\hline 2011 & 410.147 \\
\hline 2012 & 481.601 \\
\hline 2013 & 544.127 \\
\hline 2014 & 586.151 \\
\hline 2015 & 966.532 \\
\hline 2016 & 1.030 .557 \\
\hline 2017 & 1.237 .506 \\
\hline \% Variación & $202 \%$ \\
\hline
\end{tabular}

Fuente: Elaboración propia en base a datos proporcionados por la Dirección de Programación y Planificación del Ministerio de Salud de Misiones, Argentina.

Finalmente, en la Tesis de Maestría desarrollada por Almua (2018), se destacan las características que definen al modelo de gestión por resultados en el Hospital Escuela de Agudos Dr. Ramón Madariaga:

- La apuesta de la Reforma apuesta a la descentralización administrativa y a la eficiencia.

- En el régimen de sus contrataciones, el Hospital suple las normativas comunes a los demás efectores por medio de Leyes que le garantizan mayor rapidez y eficiencia en la toma de decisiones y el manejo de los recursos públicos. En el caso del Hospital Escuela de Agudos, el régimen de contrataciones resulta distintivo, en tanto que la Ley 
$\mathrm{EM}$

que crea el Parque de Salud (XVII No 70 ) en su artículo $5^{\circ}$ faculta al Poder Ejecutivo a fijar el régimen de contrataciones del Parque y a aplicar supletoriamente lo fijado en las leyes VII - $\mathrm{N}^{\mathrm{o}} 11$ (Antes Ley 2303 de Contabilidad) y X - $\mathrm{N}^{\mathrm{o}} 4$ (Antes Ley 83 de Obras Públicas). De esta manera, si bien el artículo $\mathrm{N}^{\circ} 8$ del Decreto 83/12, que reglamenta a la Ley XVII $N^{\circ} 70$, establece por regla general a la Licitación Pública, también habilita a recurrir a la contratación de los insumos, bienes o servicios necesarios previo concurso de precios y/o antecedentes. Para ello se deberán cursar invitaciones a por lo menos tres proveedores, cuyas ofertas serán analizadas por un Comité de Compras de la Fundación y aconsejará a la Dirección de Administración para la tramitación de la contratación.

- En comparación a los demás hospitales locales que aún conviven con el modelo tradicional o burocrático, el Hospital Escuela registra plazos administrativos en los procesos de compras muchos menores, lo que le permite mejorar las condiciones de negociación del efector.

- La reforma ha significado un cambio en la Organización, redirigiéndola desde los procesos hacia los resultados.

- El gerenciamiento del Hospital es de tipo privado. Se Admite liquidar a su personal adicional por producción, mayor prestación y/o mayor responsabilidad. Únicamente contrata personal temporario.

- Se destaca una estructura simple, en comparación a los demás efectores de la provincia, encontrándose en la cumbre una Fundación (quien administra y gobierna), seguido por el Parque de la Salud (con sus direcciones y departamentos) y, finalmente, los hospitales y efectores dependientes.

\section{Contexto sanitario en la de la Provincia de Misiones (Argentina) y la VII Región Sanitaria (Itapúa) de Paraguay}

La dimensión social relacionada a la salud se constituye en uno de los núcleos duros de las políticas sociales, por tener un fuerte impacto entre las personas al enmarcarse en el ámbito del derecho a la vida (Ribeiro Nogueira, 2018). En este ámbito específico de salud, el término "Equidad en Salud" se define como la ausencia de diferencias (o desigualdades) injustas, innecesarias y evitables en el estado de salud de los individuos, poblaciones o grupos que tienen diferentes características socioeconómicas, demográficas o geográficas.

Reconocer las similitudes y divergencias entre los sistemas locales de salud es un primer paso para una futura cooperación en regiones de frontera (Ribeiro Nogueira, 2018), ya que los resultados pueden convertirse en pilares para los respectivos estados, pudiendo ser aprovechados para la construcción de políticas y programas específicos.

Argentina y Paraguay son dos países limítrofes, que se encuentran integrados comercialmente a través del Mercosur, pero que todavía presentan grandes diferencias socioeconómicas.

Por parte del gobierno argentino, la existencia y ejecución de servicios sanitarios para ciudadanos de otros países del Mercosur no constituye un objetivo explícito para su Ministerio de Salud, no existe una designación y elaboración de instrumentos para las regiones de frontera y las provincias no poseen órganos específicos de salud en la frontera por ser una competencia federal (Instituto Social del Mercosur, 2018).

En Paraguay, por su parte, la disponibilidad de datos referentes a la salud y acceso de la población a los servicios en las ciudades de Encarnación (departamento de Itapúa) son insuficientes en las páginas oficiales. En lo que refiere al acceso de servicios de salud entre 
ciudades del Mercosur, varias fuentes periodísticas resaltan la tendencia de paraguayos que acuden en busca de servicios de salud hacia Argentina y Brasil (Instituto Social del Mercosur, 2018).

Del informe publicado por la Organización Mundial de la Salud acerca de los Indicadores Básicos y la Situación de Salud en la Américas en 2018, se pueden resaltar las desigualdades que derivan de la contraposición de dos sistemas de salud divergentes. Esto se puede ver reflejado en la Tabla 5:

Tabla 5: Indicadores Básicos. Argentina-Paraguay. 2018.

\begin{tabular}{|c|c|c|c|c|}
\hline & Descripción & Año & Argentina & Paraguay \\
\hline \multirow{4}{*}{$\begin{array}{c}\text { Indicadores } \\
\text { Demográficos - } \\
\text { Socioeconómicos }\end{array}$} & Esperanza de vida al nacer & 2018 & 76,9 & 73,3 \\
\hline & Tasa global de fecundidad & 2018 & 2,3 & 2,4 \\
\hline & Ingreso Nacional Bruto (US\$ per cápita - valor ppp) & 2017 & 20270 & 9180 \\
\hline & Índice de concentración de GINI & 2016 & 42,4 & 47,9 \\
\hline \multirow{6}{*}{$\begin{array}{l}\text { Indicadores del estado } \\
\text { de salud }\end{array}$} & Tasa de mortalidad infantil & 2016 & 9,7 & 13,7 \\
\hline & Tasa de mortalidad de menores de 1 año (neonatal) & 2016 & 6,5 & 9,5 \\
\hline & Razón de mortalidad materna & 2016 & 33,7 & 86,4 \\
\hline & Casos reportados de Dengue & 2017 & 557 & 1832 \\
\hline & Diagnóstico de VIH (Tasa 100.000 hab) & & $12,0^{(2015)}$ & $21,4^{(2016)}$ \\
\hline & Incidencia de Tuberculosis (100.000 hab) & 2015 & 22,1 & 35,4 \\
\hline \multirow{2}{*}{$\begin{array}{c}\text { Indicadores de cobertura } \\
\text { de servicios }\end{array}$} & $\%$ Partos hospitalarios & 2016 & 99,6 & 97,6 \\
\hline & Cobertura (\%) de la inmunización frente al sarampión en niños de 1 año & 2017 & 90 & 80 \\
\hline \multirow{4}{*}{$\begin{array}{l}\text { Indicadores del Sistema } \\
\text { de Salud }\end{array}$} & Médicos (densidad cada 10.000 hab) & 2017 & 39,6 & 2,4 \\
\hline & Gasto público en salud (\% del PIB) & 2015 & 4,9 & 4,2 \\
\hline & Gasto privado en salud (\% del PIB) & 2015 & 1,9 & 3,6 \\
\hline & Gasto de bolsillo en salud como \% del GTS & 2015 & 17,6 & 35,4 \\
\hline
\end{tabular}

Fuente: Elaboración propia en base a informe "Indicadores Básicos. Situación de Salud en las Américas. 2018" de la OPS/OMS.

De los datos presentados se observa que Argentina presenta mayor esperanza de vida al nacer, menor tasa de mortalidad, mayor número de médicos y mayor gasto público en salud. Por su parte, Paraguay, además de las desventajas en los indicadores mencionados, presenta casi dos veces más el gasto de bolsillo en salud. Esto último es importante resaltar, en tanto representa la baja participación del Estado en las políticas de Salud y los altos costos que le representan al paciente paraguayo el acceso a la atención sanitaria en su país. De esta manera, se refuerza la idea de que las personas que atraviesan la frontera se ven motivados no solo en búsqueda de atención de calidad, sino que además la gratuidad en el acceso que garantiza Argentina.

En las Ciudades de Frontera Posadas (Argentina) - Encarnación (Paraguay) se observan una serie de características compartidas que las integran de modo inédito en una conurbación transfronteriza: el desarrollo de proceso de conurbación, la habilitación del puente Internacional San Roque González de Santa Cruz que conecta ambas ciudades, la proximidad, el desarrollo de interacciones locales y el encontrarse entre relaciones de competencia y cooperación (BRITES, 2018).

Siguiendo a Brites (2018), el conjunto de características que ambas ciudades

[...], pueden ser interpretadas a partir del generalizado modelo de ciudades gemelas; ciudades donde a pesar de no contar con un gobierno común, las relaciones económicas, culturales, de vecindad y complementariedad 
$\mathrm{EM}$

producen impactos en una ciudad y viceversa, generando interdependencias, mutuos condicionamientos y una forma específica de articulación interurbana (BRITES, 2018, p. 2).

No obstante ello, durante los últimos años, Posadas se ha consolidado por su sistema sanitario público-privado como centro urbano regional de referencia para los estudios de alta complejidad tanto para residentes del interior provincial como para ciudadanos de Encarnación y otras ciudades adyacentes (BRITES, 2018).

Se pueden destacar tres aspectos que profundizan las desigualdades de salud en la frontera: la composición del sistema público de salud en ambos lados de la frontera, la situación de cobertura médica y el gasto en salud realizado por los respectivos Estados.

La Tabla 5 expone las estadísticas hospitalarias más significativas en cuanto a la desigualdad en la composición de los sistemas públicos de salud en la frontera Misiones (Argentina) y la VII Región Sanitaria (Itapúa) de Paraguay para el año 2017.

Tabla 6: Principales indicadores sanitarios. Año 2017

\begin{tabular}{|c|c|c|}
\hline \multirow[t]{2}{*}{ Principales indicadores sanitarios } & \multicolumn{2}{|c|}{2017} \\
\hline & $\begin{array}{c}\text { VII RS } \\
\text { Itapúa } \\
\text { (Paraguay) }\end{array}$ & $\begin{array}{c}\text { Misiones } \\
\text { (Argentina) }\end{array}$ \\
\hline Población estimada & 592.017 & 1.215 .029 \\
\hline $\begin{array}{l}\mathrm{N}^{\circ} \text { Total de Establecimientos de Salud del Ministerio de } \\
\text { Salud }\end{array}$ & 109 & 427 \\
\hline $\begin{array}{l}N^{\circ} \text { Total de Establecimientos con Internación del Ministerio } \\
\text { de Salud }\end{array}$ & 20 & 46 \\
\hline $\mathrm{N}^{\circ}$ de Camas (Ministerio de Salud) & 460 & 1.606 \\
\hline $\mathrm{N}^{\circ}$ de Camas por 1.000 hbtes. (Ministerio de Salud) & 0,8 & 1,3 \\
\hline $\mathrm{N}^{\circ}$ Hospitales de Alta Complejidad & 1 & 6 \\
\hline
\end{tabular}

Fuente: Elaboración propia en base a: Boletín de Indicadores básicos de Salud 2018, Paraguay (OPS-OMS) y Ministerio de Salud Pública de la Provincia de Misiones, Dirección de Programación y Planificación del Ministerio de Salud de la Provincia de Misiones, Estadísticas Hospitalarias Año 2017.

Con poco más del doble de población, la Provincia de Misiones (Argentina) cuenta con tres veces más de total de establecimientos de salud dependientes del Ministerio público (incluyendo hospitales y centros de atención primaria). Respecto del número de camas, Misiones supera en 3,5 veces la cantidad declarada en la VII Región Sanitaria de Paraguay. Por otra parte, el número de hospitales de alta complejidad también pone en ventaja a Misiones, quien concentra en su ciudad capital a tres de los seis efectores. Finalmente, se observa que el número de camas en la VII Región Sanitaria de Itapúa llega a 0,8 camas cada mil habitantes, lo que es considerado insuficiente para cubrir la demanda (Ministerio de Salud Pública y Bienestar Social de Paraguay, 2012).

Una de las principales consecuencias de estas desigualdades sanitarias en la frontera se refleja en la presencia de pacientes paraguayos que cruzan la frontera en busca de atención médica. La razón por la cual los pacientes paraguayos buscan atención sanitaria en la ciudad de Posadas se debe a "la excelencia de los servicios de salud, al suministro de medicamentos y a la existencia de servicios de mayor nivel de complejidad" (Ribeiro Nogueira, 2018, p. 69).

Para poder avanzar hacia la universalidad del acceso a la salud en zonas de frontera, es 
EM

QUESTÃO

V.13 N. $02 \downarrow 2020$

pág. $164-177$

necesario "definir e implementar políticas y acciones con un enfoque multisectorial" que logren abordar los determinantes sociales de la salud y fomenten el compromiso de toda la sociedad, en pos de promover la salud y bienestar general (SERAFINI y ZAVATTIERO, 2018)

\section{Consideraciones finales}

Las ciudades fronterizas en estudio, denotan grandes asimetrías en lo que a servicios de salud refiere. Por un lado, la última década se ha caracterizado por un crecimiento significativo de la oferta de salud de Misiones, lo que ha significado una alta inversión pública tanto en infraestructura, recursos humanos e insumos por parte del Gobierno Provincial. Por su parte, la lenta recuperación del sector salud en Paraguay ha contribuido a que gran parte de la población fronteriza recurra a los hospitales públicos misioneros en busca de atención médica, bien sea por cuestiones de distancia, de costo o de beneficios derivados de poseer nacionalidad argentina.

La última década ha significado para la Provincia de Misiones, Argentina, un cambio trascendental en lo que refiere a su política sanitaria pública. La puesta en marcha del Hospital Escuela de Agudos Dr. Ramón Madariaga representó un cambio de paradigma y una reforma en búsqueda de asegurar mayor eficiencia y calidad en sus servicios. Sin lugar a dudas, las transformaciones acaecidas arrojan indicadores sanitarios sumamente positivos, colaborando de esta manera al objetivo provincial de fortalecimiento de la salud pública.

Ahora bien, una de las principales consecuencias de esta modernización ha sido también un aumento considerable de la demanda de servicios de salud, no solo de los misioneros, sino también de pacientes de zonas fronterizas que llegan en busca de atención médica. Es por ello que resulta necesario por parte de ambos Gobiernos coordinar acciones de políticas públicas sanitarias en las zonas fronterizas, que tiendan a desvanecer las asimetrías que aun hoy ponen en contacto dos sistemas divergentes de atención a la salud pública.

Las desigualdades en la zona fronteriza del Mercosur en materia de salud, caracterizadas por la consolidación de sistemas sanitarios diferentes y fragmentados, disparidades en los niveles de cobertura y desigual porcentaje en el componente del gasto de bolsillo, tienden a profundizar las inequidades entre los países miembros. Particularmente, en la región fronteriza Misiones (Argentina) - Itapúa (Paraguay) las condiciones de desigualdad e inequidad permanecen en el tiempo.

Ante esta situación de considerables desigualdades, resulta necesario evaluar en qué medida las mismas son inequitativas, a fin de colaborar en la instrumentación de políticas públicas fronterizas tendientes a disminuirlas y así poder brindar a la población un servicio de salud más eficiente y resolutivo frente a situaciones no deseadas.

\section{Referencias:}

ALMUA Florencia Itati y ALIPRANDINI Antonella. La Problemática de la Integración Sanitaria en la Frontera Posadas, Argentina - Encarnación, Paraguay. Seminario. III Seminario Internacional de los Espacios de Frontera (III GEOFRONTERAS). UNI. Paraguay. Itapúa. 2015.

ALMUA, Florencia Itati. La Reforma del Sector Público de Salud en la Provincia de Misiones y su impacto en la atención a pacientes de frontera. $1^{\circ}$ Jornada de Jóvenes Investigadores. 
EM

QUESTÃO

V.13 N. $02 \downarrow 2020$

pág. $164-177$

Facultad de Humanidades y Ciencias Sociales. UNaM. Misiones, Argentina. 2016.

ALMUA, Florencia Itati. Las reformas en los modelos de gestión de los hospitales de alta complejidad en la provincia de misiones y su impacto en los indicadores de eficiencia hospitalaria y administrativa. Periodo 2007-2016. Posadas: Facultad de Ciencias Económicas de la Universidad Nacional de Misiones, 2018. 110 p. (Tesis de Maestría en Gestión Pública).

BRITES, Walter Fernando. ¿Ciudades gemelas? Posadas, Argentina y Encarnación, Paraguay en perspectiva sociourbana. Estudios fronterizos, 19, e020. Epub 11 de diciembre de 2018. https://dx.doi.org/10.21670/ref.1820020

ESSAYAG, Sebastián. El Modelo de Gestión por Resultados en el Sector Público Argentino. Jornadas de reflexión académica en Ciencias Sociales. Universidad de Belgrano, Facultad de Derecho y Ciencias Sociales. Argentina. Octubre 2000.

ESTÉVEZ, Alejandro M. Una comparación entre la teoría del Nuevo Management Público y la gestión de la Calidad Total. En: La reforma managerialista del Estado, compilado por Alejandro Estévez, Ediciones Cooperativas, Buenos Aires, 2001. Disponible en:http://panel.inkuba.com/sites/2/archivos/NMP\%20y\%20Teoria\%20de\%201a\%20calidad\%2 0total\%20estevez.PDF Fecha última consulta: 08/02/2020.

ESTÉVEZ, Alejandro M. El Nuevo Management Público luego de la reforma del Estado en Argentina. 2003. Disponible en: http://panel.inkuba.com/sites/2/archivos/EL\%20NMP\%20luego\%20de\%20la\%20reforma\%20 argentina\%202003.pdf Fecha última consulta: 08/02/2020.

ESTÉVEZ, Alejandro M. y BLUTMAN Gustavo. El modelo burocrático inacabado después de las reformas de los 90: ¿Funcionarios, Gerentes o Sobrevivientes? Revista Venezolana de Gerencia. 2005. Disponible en: http://panel.inkuba.com/sites/2/archivos/revista\%20venezolana\%20de\%20gerencia\%20blutm an\%20estevez.PDF Fecha última consulta: 08/02/2020.

ETORENA, A. C. y FREAZA, J. C. Historia de Posadas. Volumen II: Los barrios, lugares y servicios públicos, educación, cultura, iglesias, salud, justicia y seguridad, economía, comercio e industria. Ed. Talleres Gráficos de EXTRA Impresos; Av. Ituzaingó 2942; Posadas-Misiones, Argentina; Pág. 310-311. 2011.

GÓNGORA Norberto. y SPADAFORA Santiago. GESTIÓN DEL CAMBIO ORGANIZACIONAL. Buenos Aires, 2003.

INSTITUTO SOCIAL DEL MERCOSUR. Políticas Sociais no MERCOSUL - Estrutura dos organismos públicos de oferta dos serviços sociais. Assunção, 2018. 128 páginas. ISBN: 97899967-863-1-0. Edición impresa. 2018.

MINISTERIO DE SALUD PÚBLICA Y BIENESTAR SOCIAL DE PARAGUAY. Análisis de situación de salud. Eje Sur. Itapúa-Ñeembucú-Misiones. República del Paraguay. 2012. Disponible en: https://es.slideshare.net/Palp/analisis-de-situacion-de-salud-paraguay-eje-surindicadores Fecha última consulta: 25/11/18. 
EM

QUESTÃO

V.13 N. $02 \downarrow 2020$

pág. 164-177

RIBEIRO NOGUEIRA, Vera Maria. A dimensão social do Mercosul em espaços fronteiriços: assimetrias e convergências na atenção à saúde. Revista MERCOSUR de políticas sociales ISSN 2523-0891 (impreso) Vol 2: 2018 : pp. 60-73 - doi: 10.28917/ism.2018-v2-60. 2018.

SERAFINI, Verónica y ZAVATTIERO, Claudina. La protección social en el Paraguay; avances y desafíos para la garantía de derechos. Revista MERCOSUR de políticas sociales ISSN 2523-0891 (impreso) Vol 2 : 2018 : pp. 173-191 - doi: 10.28917/ism.2018-v2-173. 2018.

VELAZCO, Omar Luis. La dinámica socio-laboral del tránsito vecinal fronterizo. El caso Posadas (Argentina) - Encarnación (Paraguay). Informe OIM, Buenos Aires. 2000.

\section{Nota biográfica:}

Florencia Almúa - docente-investigadora en el Proyecto FCE-UNaM 16E-179 "Transfrontera Sur II. Procesos de metropolizacion binacional transfronteriza en el Cono Sur. PosadasEncarnacion, 2016 -2018”. 